\title{
Scaling law and universal drop size distribution of coarsening in conversion-limited phase separation
}

\author{
Chiu Fan Lee $\odot^{*}$ \\ Department of Bioengineering, Imperial College London, South Kensington Campus, London SW7 2AZ, United Kingdom
}

(Received 7 August 2021; accepted 21 October 2021; published 28 October 2021)

\begin{abstract}
Phase separation is not only ubiquitous in diverse physical systems, but also plays an important organizational role inside biological cells. However, experimental studies of intracellular condensates (drops with condensed concentrations of specific collections of proteins and nucleic acids) have challenged the standard coarsening theories of phase separation. Specifically, the coarsening rates observed are unexpectedly slow for many intracellular condensates. Recently, Folkmann et al. [Science 373, 1218 (2021)] argued that the slow coarsening rate can be caused by the slow conversion of a condensate constituent between the state in the dilute phase and the condensate state. One implication of this conversion-limited picture is that standard theories of coarsening in phase separation (Lifshitz-Slyozov-Wagner theory of Ostwald ripening and drop coalescence schemes) no longer apply. Surprisingly, I show here that the model equations of conversion-limited phase separation can instead be mapped onto a grain growth model in a single-phase material in three dimensions. I further elucidate the universal coarsening behavior in the late stage using analytical and numerical methods.
\end{abstract}

DOI: 10.1103/PhysRevResearch.3.043081

\section{INTRODUCTION}

Phase separation is a ubiquitous phenomenon in nature: from the separation of quark matter with distinct baryon densities in the early universe [1,2], to the everyday occurrence of dew and fog on Earth today. Besides being integral to our understanding of diverse physical systems, phase separation also plays an important organizational role in living systems: Many protein-nucleic acid condensates (i.e., drops with condensed concentrations of specific collections of proteins and nucleic acids) exist intracellularly [3,4]. This recent revelation led to an intense interest of cellular phase separation from cell biologists and biophysicists [5-8]. One of the key outstanding questions in this emergent field is as follows: Why do the coarsening rates of many condensates observed in cells seem negligible?

Coarsening of condensates refers to the evolution from an emulsion of polydisperse condensates to a single condensate coexisting with the dilute phase, as dictated by thermodynamics. Coarsening in the late stage can take on two forms: (i) the transfer of material from small drops to big drops through the dilute phase in a process known as Ostwald ripening, and (ii) the coalescence of diffusing drops when they encounter each other [8]. To account for the slow condensate coarsening rates observed in experiments, various proposals have been advocated: Driven chemical reactions that convert constituent proteins between a soluble form and a phase separating form can counter Ostwald ripening [9-11], and intracellular viscoelastic networks (e.g., the cytoskeletal

\footnotetext{
*c.lee@imperial.ac.uk

Published by the American Physical Society under the terms of the Creative Commons Attribution 4.0 International license. Further distribution of this work must maintain attribution to the author(s) and the published article's title, journal citation, and DOI.
}

networks) can limit the growth of condensates through mechanical suppression [12-15], and render the condensates' dynamics subdiffusive, which slows down coalescence [16].

More recently, drawing inspiration from the rugged energy landscape picture that explains the slow elongation rates observed in amyloid fibrillization [17-19], a conversion-limited scheme was proposed to model the coarsening dynamics of $\mathrm{P}$ granules - a type of cellular condensates found in the germ cells of the nematode Caenorhabditis elegans [20]. Indeed, in many in vitro amyloid fibrillization experiments with no driven chemical reactions, no viscoelastic networks limiting fibrillar growth, and no subdiffusive behavior, unexpectedly slow elongation rates are nonetheless observed [21-25]. To rationalize these findings, a rugged energy landscape picture was proposed in Ref. [17], in which the many local free-energy minima arise from the various suboptimal conformations that a fibrillizing protein can be stuck in before achieving the minimal free-energy state, which corresponds to the fully integrated fibrillar form. The slow fibril elongation rates observed can thus be explained by the generically slow "diffusion" over a rugged energy landscape [26].

In cellular condensates, the constituents (proteins and nucleic acids) can be thought of as polymers with multiple, potentially unspecific, binding sites. Therefore, it was argued in Ref. [20] that a condensate constituent in the dilute phase also has to go through a series of local minima (e.g., a series of suboptimal binding configurations) before being fully incorporated into the condensate [Fig. 1(a)]. As a result, a corresponding rugged energy landscape picture can potentially explain the slow coarsening rates observed.

An immediate consequence of this conversion-limited picture is that the standard theories of coarsening are no longer valid. In this paper, I will first review in detail the derivation of the model equations that describe coarsening in the conversion-limited scheme as proposed in Ref. [20]. I will then show that the model equations can be mapped onto a 
(a)

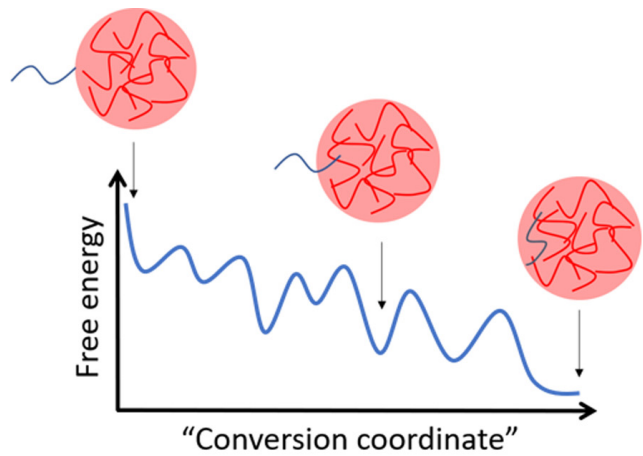

(b)
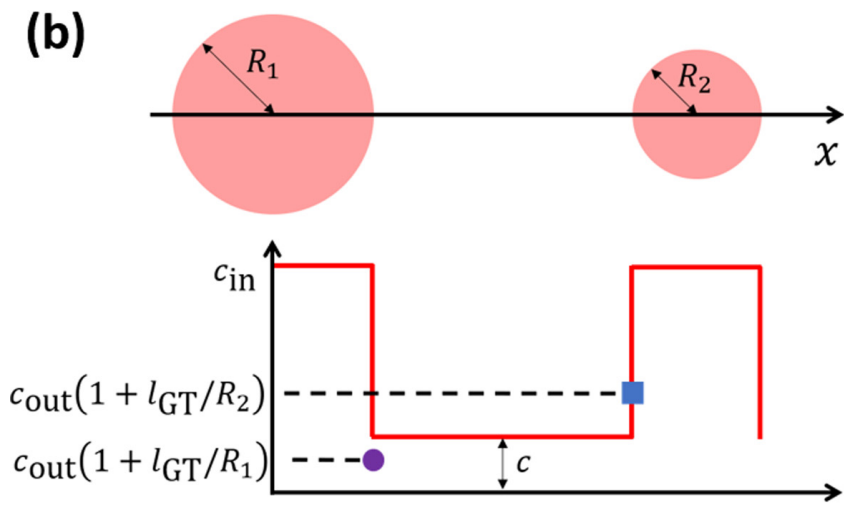

$\chi$

FIG. 1. Coarsening in the conversion-limited scheme. (a) A schematic of the rugged energy landscape that a monomer (blue) has to traverse through to become fully incorporated into the condensed drop (pink). Here, the vertical axis depicts the free energy of the blue polymer-red drop system constrained to the partially converted state along the abstracted conversion coordinate. For a polymer with multiple binding patches, the rugged energy landscape can arise from the sequential binding-unbinding events, many of them frustrated, that the polymer has to go through before being integrated into the drop. Since diffusion over a rugged energy landscape can be very slow [26], the conversion step can become rate limiting in coarsening. If this is the case, the concentration in the dilute phase, $c$, can be assumed to be constant throughout the dilute phase due to the fast molecular diffusion. (b) In a two-drop system (top figure), the concentration along the $x$ axis (red lines) according to the conversion-limited scheme is shown in the bottom figure. Due to the surface tension-induced Gibbs-Thomson relation [8], the equilibrium concentration outside the big drop (purple circle) is lower than that of the small drop (blue square). The mismatched concentrations outside the two drops lead to an outflux of material from the small drop and an influx into the big drop, resulting in the coarsening of the system. Figures adapted from Ref. [20].

grain growth model in a single-phase material in three dimensions [27]. I further elucidate the universal coarsening behavior in the late stage using analytical and numerical methods.

\section{MODEL EQUATIONS}

To proceed analytically, I will use a simplified singlecomponent model, in which a single concentration captures effectively the aggregate concentrations of the condensate constituents. In an emulsion of polydisperse drops with a uniform inner concentration $c_{\text {in }}$, the equilibrium concentration outside a drop of radius $R$ is $c_{\text {out }}\left(1+l_{\mathrm{GT}} / R\right)$, where $c_{\mathrm{out}}$ is the concentration outside a flat interface $(R \rightarrow \infty)$, and $l_{\mathrm{GT}}$ is a length scale that accounts for the Gibbs-Thomson relation arising from the surface tension-induced Laplace pressure acting on the drops [Fig. 1(b)] [8]. In the conversion-limited regime, the slow process is the conversion of a constituent molecule into and out of a drop at the interface, while molecular diffusion in the dilute phase is the fast process that renders the concentration in the dilute phase, $c$, constant throughout. By the principle of mass conservation, $c$ is given by

$$
c(t)=\frac{c_{\mathrm{tot}} V-c_{\mathrm{in}} V_{\mathrm{drops}}(t)}{V-V_{\mathrm{drops}}(t)},
$$

where $c_{\text {tot }}$ is the total solute concentration, $V$ is the volume of the system, and $V_{\text {drops }}(t)=\frac{4 \pi}{3} \sum_{i=1}^{N} R_{i}(t)^{3}$ is the total drop volume in the system with $N$ being the number of drops.

To achieve the equilibrium state of having a single drop coexisting with the dilute phase, the system inevitably coarsens. To consider the universal behavior in the late stage, I will focus exclusively on coarsening by Ostwald ripening under the conversion-limited scheme, and justify the neglect of coarsening by drop coalescence later.

The thermodynamic drive towards equilibrium is caused by the mismatch between the radius-dependent equilibrium concentrations outside the drops given by

$$
c_{\mathrm{out}}\left(1+\frac{l_{\mathrm{GT}}}{R}\right),
$$

and the concentration of the dilute phase, $c(t)$ [Fig. 1(b)]. Here, I assume that the mismatch is small so that the resulting material flux is proportional to the concentration difference. Specifically, the flux $J_{R}(t)$ into (when positive) and out of (when negative) a drop of radius $R$ is given by

$$
J_{R}(t)=\kappa^{\prime}\left[c(t)-c_{\text {out }}\left(1+\frac{l_{\mathrm{GT}}}{R(t)}\right)\right],
$$

where $\kappa^{\prime}$ is a constant of dimension (length)/(time), which is proportional to the effective adsorption (desorption) rate of a polymer into (out of) the drop caused by the mismatched boundary conditions at the drop's interface. The rate is effective in the sense that it corresponds to the statistical average of many microscopic adsorption of desorption events that occur at the interface.

In an $N$-drop system, the rates of change of the drops' radii are thus

$$
\frac{d R_{i}(t)}{d t}=\frac{J_{R_{i}}(t)}{c_{\text {in }}}=\kappa\left(\frac{1}{R_{c}(t)}-\frac{1}{R_{i}(t)}\right),
$$

for $1 \leqslant i \leqslant N$, where $\kappa \equiv \kappa^{\prime} l_{\mathrm{GT}} c_{\mathrm{out}} / c_{\text {in }}$ and

$$
R_{c}(t)=\frac{l_{\mathrm{GT}}}{c(t) / c_{\mathrm{out}}-1},
$$

which is termed the critical radius since all drops of radii below $R_{c}$ shrink, and vice versa. The set of $N$ differential equations (4) are coupled through the mass conservation condition in (1) and (5).

Surprisingly, the model equations of conversion-limited phase separation (4) can in fact be mapped onto a grain growth 
model in a single-phase material in three dimensions studied by Hillert [27]. In this mapping, the parameter $\kappa$ corresponds to the proportionality constant that relates the velocity of the advancement of a grain boundary and the local curvature of the grain boundary. Interestingly, discrepancies between actual grain growth and the Hillert's model are known and are caused by the mean-field assumption when calculating the grain boundary curvature [28]. Such a deficiency does not occur in our system since the drops remain spherical at all times.

\section{CONVERSION-LIMITED VERSUS LIFSHITZ-SLYOZOV-WAGNER}

In the Lifshitz-Slyozov-Wagner (LSW) scheme, the prefactor $\kappa$ in (4) is replaced by $D l_{\mathrm{GT}} c_{\mathrm{out}} /\left[c_{\mathrm{in}} R(t)\right][8,29,30]$, where $D$ is the diffusion coefficient of the molecule in the dilute phase. Comparing these two quantities, one expects that the conversion-limited scheme is valid when the following condition is satisfied:

$$
\kappa \ll \frac{D}{\langle R(t)\rangle} .
$$

For a typical protein of linear dimension around $1 \mathrm{~nm}, D$ is of the order $10^{7} \mathrm{~nm}^{2} / \mathrm{s}$ [31]. Taking the typical condensate size to be in the order of $1000 \mathrm{~nm}$, the conversion-limited regime is expected to be valid if $\kappa \ll 10^{4} \mathrm{~nm} / \mathrm{s}$. As an example, a recent study of $\mathrm{P}$ granules in the single-cell embryonic stage estimated that the parameter $\kappa$ is of the order $1 \mathrm{~nm} / \mathrm{s}$ [20]. Therefore, the conversion-limited scheme is appropriate for that system.

\section{SCALING LAW AND UNIVERSAL SIZE DISTRIBUTION}

Having reviewed the physics underlying the conversionlimited scheme and compared the model equations to those of the LSW scheme, I will now elucidate analytically the universal behavior of coarsening in the asymptotic long-time limit. While it is unclear whether this asymptotic regime is relevant to intracellular condensates in vivo, the emergent universal physics can clearly be tested in controlled experiments, and be applicable to diverse natural, reconstituted, or synthetic phase-separating systems, such as various phase-separating polymers with polyvalent binding sites.

As noted before, the model equations can be mapped onto a grain growth model in a single-phase material in three dimensions [27], where the coarsening behavior has also been analyzed analytically. For completeness, I will present a similar derivation of the analytical results in this section, while highlighting how they differ from the predictions of the standard theories of phase separation.

As the identities of individual drops are irrelevant, I will start by focusing on the following time-dependent drop size distribution function:

$$
n(R, t)=\frac{1}{V} \sum_{i=1}^{N} \delta\left(R-R_{i}(t)\right) .
$$

Now, recall that in the asymptotic long-time limit of the LSW regime, the distribution function $n$ approaches the scal- ing form [29,32]

$$
\lim _{t \rightarrow \infty} n_{\mathrm{LSW}}(R, t)=\left[R_{c}(t)\right]^{-4} g_{\mathrm{LSW}}(z),
$$

where $z(t) \equiv R / R_{c}(t)$ and $g_{\mathrm{LSW}}$ is a dimensionless scaling function given by

$$
g_{\mathrm{LSW}}(z) \propto \frac{z^{2} \exp \left(1-\frac{3}{3-2 z}\right)}{\left(1+\frac{z}{3}\right)^{7 / 3}\left(1-\frac{2 z}{3}\right)^{11 / 3}},
$$

for $0 \leqslant z \leqslant 3 / 2$. In other words, in the long-time regime, the drop size probability distribution, once renormalized by the critical radius, is temporally invariant. I will assume that the same scale-invariant structure remains true for the conversionlimited scheme. Specifically, I will use the ansatz

$$
n(R, t)=\left[R_{c}(t)\right]^{-4} g(z(t)),
$$

where $g(\cdot)$ is a dimensionless function. In fact, the ansatz (10) is, as in the LSW scheme, an inevitable outcome of mass conservation in the system. The demonstration of this asymptotic behavior mirrors exactly that of the Lifshitz-Slyozov-Wagner theory $[27,29,32]$, and therefore will not be repeated here.

I will now calculate $\partial_{t} n$ by using first the definition of $n$ in (7) to get

$$
\begin{aligned}
\partial_{t} n(R, t) & =-\frac{\partial}{\partial R}\left[\kappa\left(\frac{1}{R_{c}(t)}-\frac{1}{R}\right) n(R, t)\right] \\
& =-\frac{\kappa}{R_{c}^{6}}\left[\frac{g}{z^{2}}+\left(1-\frac{1}{z}\right) g^{\prime}\right] .
\end{aligned}
$$

In the second equality above, I have replaced all $R$ and $n$ in the last expression by $z$ and $g$ using the ansatz (10).

Calculating $\partial_{t} n$ for a second time, but using the ansatz (10) directly instead, we get

$$
\partial_{t} n(R, t)=-\frac{1}{R_{c}^{5}} \frac{d R_{c}}{d t}\left(4 g+z g^{\prime}\right),
$$

where $g^{\prime}=d g / d z$.

Equating (12) and (11b), and then separating the $R_{c}(t)$ and $t$ on one side, and $g(z)$ and $z$ on the other, we have

$$
\frac{R_{c}}{\kappa} \frac{d R_{c}}{d t}=A=\frac{g / z^{2}+(1-1 / z) g^{\prime}}{4 g+z g^{\prime}},
$$

where $A$ is a constant to be determined.

From the first equality, we can see that the critical radius $R_{c}$ scales as $t^{1 / 2}$. Therefore, $R_{c}$ increases much faster than the LSW scaling law: $R_{c}^{\mathrm{LSW}}(t) \sim t^{1 / 3}$. Since the scaling law corresponding to coalescence-driven coarsening is identical to that of the LSW scheme [33,34], the increase in the power law in the conversion-limited scheme justifies the neglect of coalescence-driven coarsening in the late stage.

Note that a generalized LSW scheme that accounts for a concentration-dependent mobility term can also lead to modified scaling laws [35]. However, the models considered there always lead to a slowing down of coarsening compared to the LSW scheme.

Focusing now on the drop sizes, solving the differential equation from the second equality in (13) leads to the universal normalized distribution,

$$
g(z) \propto \frac{z \exp \left(-\frac{6}{2-z}\right)}{(2-z)^{5}},
$$



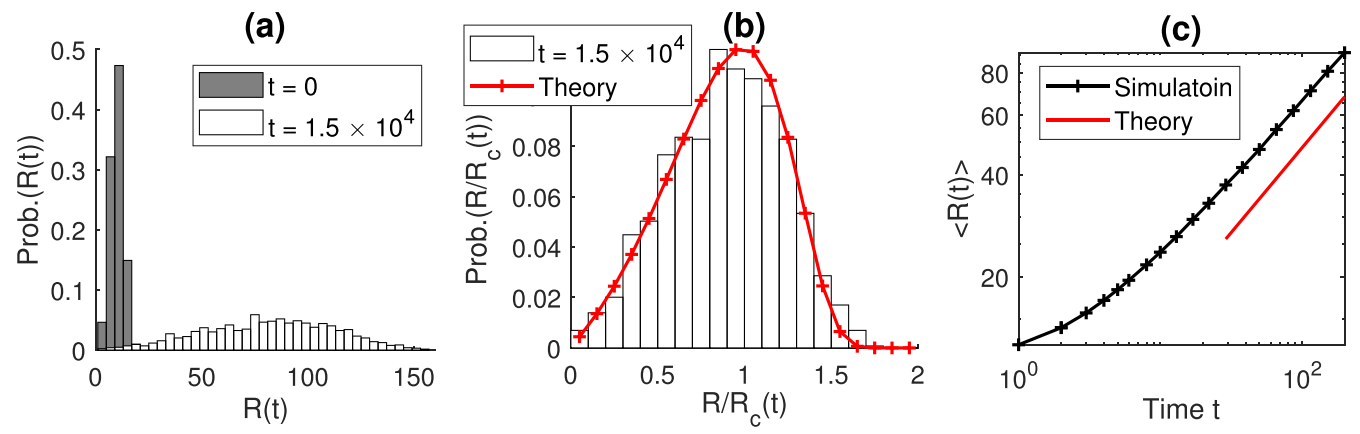

FIG. 2. Simulation vs theory. (a) The broadening of the drop size distribution that starts with a Gaussian distribution (mean $=10$, standard deviation $=3$ ), but truncated so that the random variable is always positive (gray). The other parameters are $N=5 \times 10^{5}, \kappa=1, c_{\text {out }}=1$, $c_{\text {in }}=20, c_{\text {tot }}=c_{\text {in }}+99 c_{\text {out }}\left[1+\langle R(t=0)\rangle^{-1}\right]$. Note that the late-stage universal behaviors discussed here are not dependent on the choice of the parameters. (b) The drop size distribution (at $t=1.5 \times 10^{5}$ ) and (c) the temporal evolution of $\langle R(t)\rangle$ show good agreement with the theoretical predictions (14) and (15), respectively.

for $0 \leqslant z \leqslant 2$. By calculating the average of the distribution, we find the following,

$$
\langle R(t)\rangle=\frac{24}{27} R_{c}(t) \sim t^{1 / 2},
$$

which is again different from the LSW theory: $\langle R(t)\rangle=$ $R_{c}^{\mathrm{LSW}}(t)$. Note that the expressions in (14) and (15) are equivalent to those of grain size distribution and growth rate in the late stage found in Ref. [27]. Incidentally, the $t^{1 / 2}$ scaling law (15) also coincides with the scaling law expected from the coarsening in a nonconserved system undergoing phase ordering [36,37].

Figure 2 shows the verifications of all theoretical predictions by numerically solving the model equations (1), (4), and (5). The details of the numerical procedure are given in the Appendix.

\section{DISCUSSION AND OUTLOOK}

In summary, I have discussed the physics underlying the late-stage coarsening of a phase-separating system under the conversion-limited scheme, and elucidated the scaling law and universal drop size distribution in this regime. Besides the change of the coarsening power law, the conversion-limiting scheme is also arguably more universal compared to the LSW scheme due to the uniform concentration in the dilute phase because (i) the spatial correlation of drops is irrelevant and thus the "mean-field" scenario considered here is exact [38], and (ii) the universal behavior is independent of the spatial dimension $[39,40]$.

Referring back to the condition (6) under which the conversion-limited regime is valid, it is clear that as the average drop size grows $(\langle R(t)\rangle \rightarrow \infty)$, the system will transition into the LSW scheme eventually. In other words, the asymptotic results described here are strictly speaking only applicable to some intermediate-scaling regime. However, depending on the model parameters, this intermediate-scaling regime can be extremely long. For instance, using the parameters estimated in an in vivo study of $\mathrm{P}$ granules in the one-cell embryo [20], one finds that the transition from the conversion-limited regime to the LSW regime occurs when $\langle R\rangle \approx 10^{7} \mathrm{~nm}$, which corresponds to an intermediate-scaling regime that spans over 10000 years.

A final surprise here is that the universal behavior is uncovered in a system that is, while clearly motivated by a biological system, purely thermal. This is in contrast to, e.g., another class of living matter-motivated systems-polar active matter, in which diverse novel universality classes emerge from the nonequilibrium nature of the systems [41-48]. Overall, this work highlights once again that biological systems constitute a fertile ground for novel physics [49].

In terms of outlook, one potentially interesting direction will be to consider the impact of having multiple coexistence phases [50-53] on the coarsening behavior. Although for the standard LSW theory, having multiple phases does not seem to influence the asymptotic scaling behavior [54,55], it would be interesting to see whether the growth of drops of distinct phases in the intermediate stage will be affected by potential competition over shared components.

Another interesting direction will be the study of how the universal behavior of phase separation can be impacted by nonequilibrium processes in cells, which can include the active motility of the constituent components (e.g., due to molecular motors) and driven chemical reactions [e.g., adenosine triphosphate (ATP)-driven enzymatic reactions].

\section{ACKNOWLEDGMENT}

C.F.L. thanks Andrew Folkmann, Andrea Putnam, and Geraldine Seydoux for many stimulating discussions.

\section{APPENDIX: NUMERICAL PROCEDURE}

To study the temporal evolution of the drop sizes (Fig. 2), the following numerical procedure is used:

(1) Initiation. At time $t=0$, Gaussian variables (with mean $=10$, standard deviation $=3$ ) are repeatedly drawn until $N$ positive numbers are obtained. These will be the initial radii of the $N$ drops $R_{i}(t=0), i=1, \ldots, N$.

(2) Evolution. The drop sizes are updated according to the following,

$$
R_{i}(t+\Delta t)=R_{i}(t)+\Delta t \times \kappa\left(\frac{1}{R_{c}(t)}-\frac{1}{R_{i}(t)}\right),
$$


where $\Delta t=0.01$, and $R_{c}(t)$ is calculated using (5). Any $R_{i}(t)$ that drops below $10^{-3}$ will be taken out of the system. Time $t$ is then updated to $t+\Delta t$.
The model parameters used to simulate the results presented in Fig. 2 are shown in the caption. However, the universal size distribution (14) and scaling law (15) are not dependent on the choice of the parameters in the late stage.
[1] D. Schwarz, The first second of the Universe, Ann. Phys. 12, 220 (2003)

[2] J. Randrup, Phase transition dynamics for baryon-dense matter, Phys. Rev. C 79, 054911 (2009).

[3] A. A. Hyman, C. A. Weber, and F. Jülicher, Liquid-liquid phase separation in biology, Annu. Rev. Cell Dev. Biol. 30, 39 (2014).

[4] Y. Shin and C. P. Brangwynne, Liquid phase condensation in cell physiology and disease, Science 357, eaaf4382 (2017).

[5] C. Brangwynne, P. Tompa, and R. Pappu, Polymer physics of intracellular phase transitions, Nat. Phys. 11, 899 (2015).

[6] S. F. Banani, H. O. Lee, A. A. Hyman, and M. K. Rosen, Biomolecular condensates: organizers of cellular biochemistry, Nat. Rev. Mol. Cell Biol. 18, 285 (2017).

[7] J. Berry, C. P. Brangwynne, and M. Haataja, Physical principles of intracellular organization via active and passive phase transitions, Rep. Prog. Phys. 81, 046601 (2018).

[8] C. A. Weber, D. Zwicker, F. Jülicher, and C. F. Lee, Physics of active emulsions, Rep. Prog. Phys. 82, 064601 (2019).

[9] D. Zwicker, M. Decker, S. Jaensch, A. A. Hyman, and F. Jülicher, Centrosomes are autocatalytic droplets of pericentriolar material organized by centrioles, Proc. Natl. Acad. Sci. USA 111, E2636 (2014).

[10] D. Zwicker, A. A. Hyman, and F. Jülicher, Suppression of Ostwald ripening in active emulsions, Phys. Rev. E 92, 012317 (2015).

[11] J. D. Wurtz and C. F. Lee, Chemical-Reaction-Controlled Phase Separated Drops: Formation, Size Selection, and Coarsening, Phys. Rev. Lett. 120, 078102 (2018).

[12] M. Feric and C. P. Brangwynne, A nuclear F-actin scaffold stabilizes ribonucleoprotein droplets against gravity in large cells, Nat. Cell Biol. 15, 1253 (2013).

[13] R. W. Style, T. Sai, N. Fanelli, M. Ijavi, K. Smith-Mannschott, Q. Xu, L. A. Wilen, and E. R. Dufresne, Liquid-Liquid Phase Separation in an Elastic Network, Phys. Rev. X 8, 011028 (2018).

[14] K. A. Rosowski, T. Sai, E. Vidal-Henriquez, D. Zwicker, R. W. Style, and E. R. Dufresne, Elastic ripening and inhibition of liquidliquid phase separation, Nat. Phys. 16, 422 (2020).

[15] Y. Zhang, D. S. W. Lee, Y. Meir, C. P. Brangwynne, and N. S. Wingreen, Mechanical Frustration of Phase Separation in the Cell Nucleus by Chromatin, Phys. Rev. Lett. 126, 258102 (2021).

[16] D. S. W. Lee, N. S. Wingreen, and C. P. Brangwynne, Chromatin mechanics dictates subdiffusion and coarsening dynamics of embedded condensates, Nat. Phys. 17, 531 (2021).

[17] C. F. Lee, J. Loken, L. Jean, and D. J. Vaux, Elongation dynamics of amyloid fibrils: A rugged energy landscape picture, Phys. Rev. E 80, 041906 (2009).

[18] J. E. Straub and D. Thirumalai, Toward a molecular theory of early and late events in monomer to amyloid fibril formation, Annu. Rev. Phys. Chem. 62, 437 (2011).
[19] J. D. Schmit, Kinetic theory of amyloid fibril templating, J. Chem. Phys. 138, 185102 (2013).

[20] A. W. Folkmann, A. Putnam, C. F. Lee, and G. Seydoux, Regulation of biomolecular condensates by interfacial protein clusters, Science 373, 1218 (2021).

[21] A. Lomakin, D. B. Teplow, D. A. Kirschner, and G. B Benedek, Kinetic theory of fibrillogenesis of amyloid $\beta$-protein, Proc. Natl. Acad. Sci. USA 94, 7942 (1997).

[22] T. Scheibel, J. Bloom, and S. Lindquist, The elongation of yeast prion fibers involves separable steps of association and conversion, Proc. Natl. Acad. Sci. USA 101, 2287 (2004).

[23] T. Ban, M. Hoshino, S. Takahashi, D. Hamada, K. Hasegawa, H. Naiki, and Y. Goto, Direct observation of A $\beta$ amyloid fibril growth and inhibition, J. Mol. Biol. 344, 757 (2004).

[24] S. R. Collins, A. Douglass, R. D. Vale, and J. S. Weissman, Mechanism of prion propagation: Amyloid growth occurs by monomer addition, PLoS Biol. 2, e321 (2004).

[25] T. P. J. Knowles, W. Shu, G. L. Devlin, S. Meehan, S. Auer, C. M. Dobson, and M. E. Welland, Kinetics and thermodynamics of amyloid formation from direct measurements of fluctuations in fibril mass, Proc. Natl. Acad. Sci. USA 104, 10016 (2007).

[26] R. Zwanzig, Diffusion in a rough potential, Proc. Natl. Acad. Sci. USA 85, 2029 (1988).

[27] M. Hillert, On the theory of normal and abnormal grain growth, Acta Metall. 13, 227 (1965).

[28] R. Darvishi Kamachali and I. Steinbach, 3-D phase-field simulation of grain growth: Topological analysis versus mean-field approximations, Acta Mater. 60, 2719 (2012).

[29] I. Lifshitz and V. Slyozov, The kinetics of precipitation from supersaturated solid solutions, J. Phys. Chem. Solids 19, 35 (1961).

[30] C. Wagner, Theorie der Alterung von Niederschlägen durch Umlösen (Ostwald-Reifung), Ber. Bunsenges. Phys. Chem. 65, 581 (1961).

[31] R. Milo and R. Phillips, Cell Biology by the Numbers (Garland Science, New York, 2015).

[32] E. M. Lifshitz and L. P. Pitaevskii, Physical Kinetics, Course of Theoretical Physics (Butterworth-Heinemann, Oxford, UK, 1981), Vol. 10.

[33] E. D. Siggia, Late stages of spinodal decomposition in binary mixtures, Phys. Rev. A 20, 595 (1979).

[34] M. Cates, Complex Fluids: The Physics of Emulsions (Oxford University Press, Oxford, UK, 2017), Vol. 1.

[35] A. J. Bray and C. L. Emmott, Lifshitz-Slyozov scaling for latestage coarsening with an order-parameter-dependent mobility, Phys. Rev. B 52, R685 (1995).

[36] A. Bray, Theory of phase-ordering kinetics, Adv. Phys. 51, 481 (2002).

[37] P. L. Krapivsky, S. Redner, and E. Ben-Naim, A Kinetic View of Statistical Physics (Cambridge University Press, Cambridge, UK, 2010). 
[38] J. H. Yao, K. R. Elder, H. Guo, and M. Grant, Ostwald ripening in two and three dimensions, Phys. Rev. B 45, 8173 (1992).

[39] M. San Miguel, M. Grant, and J. D. Gunton, Phase separation in two-dimensional binary fluids, Phys. Rev. A 31, 1001 (1985).

[40] T. M. Rogers and R. C. Desai, Numerical study of late-stage coarsening for off-critical quenches in the Cahn-Hilliard equation of phase separation, Phys. Rev. B 39, 11956 (1989).

[41] T. Vicsek, A. Czirók, E. Ben-Jacob, I. Cohen, and O. Shochet, Novel Type of Phase Transition in a System of Self-Driven Particles, Phys. Rev. Lett. 75, 1226 (1995).

[42] J. Toner and Y. Tu, Long-Range Order in a Two-Dimensional Dynamical $X Y$ Model: How Birds Fly Together, Phys. Rev. Lett. 75, 4326 (1995).

[43] J. Toner and Y. Tu, Flocks, herds, and schools: A quantitative theory of flocking, Phys. Rev. E 58, 4828 (1998).

[44] L. Chen, J. Toner, and C. F. Lee, Critical phenomenon of the order-disorder transition in incompressible active fluids, New J. Phys. 17, 042002 (2015).

[45] J. Toner, N. Guttenberg, and Y. Tu, Swarming in the Dirt: Ordered Flocks with Quenched Disorder, Phys. Rev. Lett. 121, 248002 (2018).

[46] J. Toner, N. Guttenberg, and Y. Tu, Hydrodynamic theory of flocking in the presence of quenched disorder, Phys. Rev. E 98, 062604 (2018).
[47] L. Chen, C. F. Lee, and J. Toner, Moving, Reproducing, and Dying Beyond Flatland: Malthusian Flocks in Dimensions $d>2$, Phys. Rev. Lett. 125, 098003 (2020).

[48] L. Chen, C. F. Lee, and J. Toner, Universality class for a nonequilibrium state of matter: A $d=4-\epsilon$ expansion study of Malthusian flocks, Phys. Rev. E 102, 022610 (2020).

[49] C. F. Lee and J. D. Wurtz, Novel physics arising from phase transitions in biology, J. Phys. D 52, 023001 (2019).

[50] R. P. Sear and J. A. Cuesta, Instabilities in Complex Mixtures with a Large Number of Components, Phys. Rev. Lett. 91, 245701 (2003).

[51] W. M. Jacobs and D. Frenkel, Phase Transitions in Biological Systems with Many Components, Biophys. J. 112, 683 (2017).

[52] S. Mao, D. Kuldinow, M. P. Haataja, and A. Košmrlj, Phase behavior and morphology of multicomponent liquid mixtures, Soft Matter 15, 1297 (2019).

[53] W. M. Jacobs, Self-Assembly of Biomolecular Condensates with Shared Components, Phys. Rev. Lett. 126, 258101 (2021).

[54] C. Jeppesen and O. G. Mouritsen, Universality of ordering dynamics in conserved multicomponent systems, Phys. Rev. B 47, 14724 (1993).

[55] S. K. Das and S. Puri, Dynamics of phase separation in multicomponent mixtures, Phys. Rev. E 65, 026141 (2002). 